smoke or nitrogen dioxide exposure, in an analysis analagous to that presented in our article, no such synergy is apparent. Again, given the limited sample size and specific context of our analysis, no definitive conclusion can be drawn from these results, but it is possible that dog/endotoxin may synergise more powerfully with indoor air pollutants, relative to other exposures. If so, it may be important to understand the biology that underlies this effect.

\section{Carlsten, M. Chan-Yeung and M. Brauer}

Dept of Medicine, University of British Columbia, Vancouver, BC, Canada.
Correspondence: C. Carlsten, University of British Columbia, UBC The Lung Centre, 7th Floor, 2775 Laurel Street, Vancouver, BC, V5Z 1M9, Canada. E-mail: chris.carlsten@vch.ca

Statement of Interest: None declared.

\section{REFERENCES}

1 Carlsten C, Brauer B, Dimich-Ward H, et al. Combined exposure to dog and indoor pollution: incident asthma in a high-risk birth cohort. Eur Respir J 2011; 37: 324-330.

\title{
Interferon- $\gamma$ release assays for diagnosis of active pleural tuberculosis: a developing world perspective
}

\section{To the Editors:}

We read with interest the article by SESTER et al. [1].The results are clinically helpful, but there are some points that need to be highlighted from the perspective of the physician practising in tuberculosis (TB)-endemic areas in the developing world, where TB remains by far the commonest cause of an exudative pleural effusion [2].

Determining the aetiology of pleural effusions is a challenging problem in these clinical settings. Conventional diagnostic tests for pleural TB include microscopic examination of fluid, mycobacterial culture and histopathological examination of pleural tissue for granulomatous inflammation. However, these tests have several limitations.

The interferon- $\gamma$ release assays (IGRAs) are technically more complicated and expensive than established biomarkers and their diagnostic performance for active TB is highly variable between studies. A study comparing the cost-effectiveness of performing interferon (IFN)- $\gamma$ estimation in comparison to adenosine deaminase (ADA) for pleural effusion found that even though it was more sensitive, the cost of using IFN- $\gamma$ for detecting one additional patient was equivalent to the cost of complete TB treatment for six patients [3,4]. In developing countries where the burden of TB is high and cost is a major issue, pleural fluid IFN- $\gamma$ does not seem to be an attractive means of differentiating TB from non-TB aetiology. In fact, the World Health Organization Strategic and Technical Advisory Group for Tuberculosis (WHO STAG-TB) has not yet endorsed the use of IGRAs in resource-limited countries [5].

In contrast, pleural fluid ADA measurement has good sensitivity and specificity. It is inexpensive, simple and easy to perform and does not require any special equipment. These qualities make it an ideal inflammatory marker in resourcelimited settings where there is a high burden of TB incidence. A high diagnostic accuracy of ADA measurement has been reported in several studies. A meta-analysis by GRECO et al. [6] found that among 31 studies, which included $\sim 4,700$ patients, the pooled sensitivity was $92 \%$ and specificity was $89 \%$.
In conclusion, IGRAs, although potentially useful tools for diagnosing extrapulmonary $\mathrm{TB}$, are still not suitable for highTB burden, low-resource countries. A possible practical solution would be to use ADA measurement as the test of choice at the community level and IFN- $\gamma$ in tertiary referral institutions.

\section{K. Shah and Z. Udwadia}

Dept of Pulmonary Medicine, P.D. Hinduja National Hospital and Medical Research Centre, Mumbai, India.

Correspondence: K. Shah, Dept of Pulmonary Medicine, P.D. Hinduja National Hospital and Medical Research Centre, Veer Savarkar Marg, Mahim (W), Mumbai, Maharashtra 400016, India. E-mail: drkushal83@gmail.com

Statement of Interest: None declared.

\section{REFERENCES}

1 Sester M, Sotgiu G, Lange C, et al. Interferon- $\gamma$ release assays for the diagnosis of active tuberculosis: a systemic review and metaanalysis. Eur Respir J 2011; 37: 100-111.

2 Udwadia ZF, Sen T. Pleural effusion: an update. Current Opin Pulm Med 2010; 16: 399-406.

3 Sharma SK, Banga A. Pleural fluid interferon- $\gamma$ and adenosine deaminase levels in tuberculosis pleural effusion: a cost-effectiveness analysis. J Clin Lab Anal 2005; 19: 40-46.

4 Trajman A, Pai M, Dheda K, et al. Novel tests for diagnosing tuberculous pleural effusion: what works and what does not? Eur Respir J 2008; 31: 1098-1106.

5 Report of WHO Expert Group on use of interferon- $\gamma$ release assays (IGRAs) in tuberculosis control in low-and middle-income setting, 20-21 July, 2010. http:/ /www.who.int/tb/advisory_bodies/stag_tb_report_2010.pdf Date last accessed: June 20, 2011.

6 Greco S, Girardi E, Masciangelo R et al. Adenosine deaminase and interferon- $\gamma$ measurements for the diagnosis of tuberculosis pleurisy: a meta-analysis. Int J Tuberc Lung Dis 2003; 7: 777-786. 